\title{
Gene silencing using a conjugate comprising Tat peptide and antisense oligonucleotide with phosphorothioate backbones
}

\author{
Yoshiya Maegawa ${ }^{\mathrm{a}}$, Shinichi Mochizuki ${ }^{\mathrm{a}, *}$, Noriko Miyamoto ${ }^{\mathrm{a}}$, and Kazuo Sakurai ${ }^{\mathrm{a}, \mathrm{b}, *}$ \\ ${ }^{a}$ Department of Chemistry and Biochemistry, The University of Kitakyushu, 1-1, Hibikino, Wakamatsu-ku, Kitakyushu, Fukuoka, 808-0135, Japan. \\ ${ }^{b}$ CREST, Japan Science and Technology Agency, 4-1-8, Honcho, Kawaguchi-shi, Saitama, 332-0012, Japan.
}

\section{ARTICLE INFO}

\section{ABSTRACT}

Article history:

Received

Revised

Accepted

Available online

Keywords:

Antisense oligonucleotide

Tat peptide

Conjugate

Click Chemistry

\begin{abstract}
Antisense Oligonucleotides (ASOs) have a great therapeutic potential for the modulation of gene expression because of the high specificity. The major obstacles for clinical application are enzymatic degradation and low uptake into cells in vivo. In this study, we prepared the conjugate comprising Tat peptide and ASO with phosphorothioate linkages in a simple manner; azide alkyne Huisgen cycloaddition using a copper catalyst. The obtained conjugate showed a high stability in serum, compared with the conjugate with phosphodiester linkages. The conjugates with antisense for c-myb that is transcriptional factor concerning cell growth inhibited the cell proliferation in a dose dependent manner sequence-specifically. These findings suggest Tat-mediated ASOs delivery is useful for the treatment of various diseases.
\end{abstract}

* Corresponding author. Tel.: +81-093-695-3203; fax: +81-093-695-3390; e-mail: mochizuki@kitakyu-u.ac.jp

* Corresponding author. Tel.: +81-093-695-3294; fax: +81-093-695-3390; e-mail: sakurai@kitakyu-u.ac.jp 
Antisense oligonucleotides (ASOs) are single-stranded nucleic acids consisting of 15-25 nucleotides in length, which bind to the target RNA sequence specifically, resulting in interference with gene expression. ASOs can modulate gene expression by several mechanisms including RNase $\mathrm{H}$-mediated degradation of the target $\mathrm{RNA}^{1,2}$ or skipping of the targeted exon and altering the RNA and protein sequence ${ }^{3}$. One of the major obstacles for clinical application of ASOs is enzymatic degradation in vivo. From first introduction by Zamecnik et al. ${ }^{4}$, chemical modifications to the backbone have been at the center of research interest for improvement of stability in vivo. Among chemical modifications, phosphorothioate (PS) backbone that replaced one of the nonbridging oxygen atoms in the phosphodiester backbone with a sulfur atom is termed the first generation of oligonucleotide modifications and reported to show a high stability in serum ${ }^{5}$. Despite the benefit, there is room for consideration of cellular uptake. Thus, the efficient and non-toxic delivery systems have been developed by many groups, including liposomes and cationic polymer complexes. Among the various proposed delivery carriers, cell-penetrating peptides (CPPs) have much attention as the most promising carrier for internalization into cells ${ }^{6}$.

Ryser and his colleagues first demonstrated that conjugation of cationic peptides to anticancer drugs can improve cellular uptake in vitro and in vivo ${ }^{7}$. Most CPPs, small and basic amino acids-rich peptide as shown in HIV-1 Tat protein, are found to have the ability to translocate across cellular plasma membranes ${ }^{8,9}$. Therefore they are often used by covalent conjugation with drugs or drug-embedded carriers. In case of conjugation with oligo nucleotides (ODNs), a disulfide, ester and peptide bonds are employed. Among the conjugations, a disulfide bond between peptide with cysteine residue and maleimide-modified ODNs is largely advantageous because a disulfide linkage is dissociated in the reducing milieu, resulting in release of ODN from peptide ${ }^{10}$. Although there are many reports concerning the synthesis of peptide-ODN conjugates (POCs) comprising a peptide and an ODN with phosphodiester backbones, only few papers describe the preparation of POCs with PS backbones (POCPs) ${ }^{11}$. Since the ODNs with PS backbones can induce a side react with a peptide bearing a disulfide reactive functional group, it is difficult to purify and evaluate the characterization of POCPs linked at the desired sites. Azhayev and his colleagues reported well-characterized POCPs using a PS-ODN with a mercaptoalkyl group and a peptide with a pyridyl disulfide function ${ }^{12}$. "Click Chemistry" is a concept of chemical reaction introduced by K. B. Sharpless at el. in $2001^{13}$. Azide alkyne Huisgen cycloaddition using a copper catalyst is one of the most popular reactions and can be proceeded in benign reaction conditions and give high yields ${ }^{14}$. In this study, we synthesized POCPs with a simple reaction, where the POCPs comprise one of the major CPPs, Tat peptide, and PS-modified ASO (PS-ASO) for c-myb that is transcriptional factor concerning cell growth ${ }^{15}$, and examined the inhibition effect of cell growth by treatment with POCPs.

We prepared PS-ASO with alkyne group at 5'-terminus and Tat peptide with azide group at C-terminus (Figure 1A), and mixed the both compounds at various molar ratios with copper catalyst for the optimization of reaction condition. After the reaction, we soon added a chelate agent ethylenediaminetetraacetic acid (EDTA) to prevent the aggregations because PS-ASOs are considered to be easy to crosslink via cupper molecules. To confirm the conjugation, the reaction mixtures were separated by $15 \%$ polyacrylamide gel electrophoresis (PAGE). When Tat peptide was mixed with PS-ASO at the same molar ratio, a new band appeared at a larger molecular weight than PS-ASO (Figure 1B). As Tat peptide was

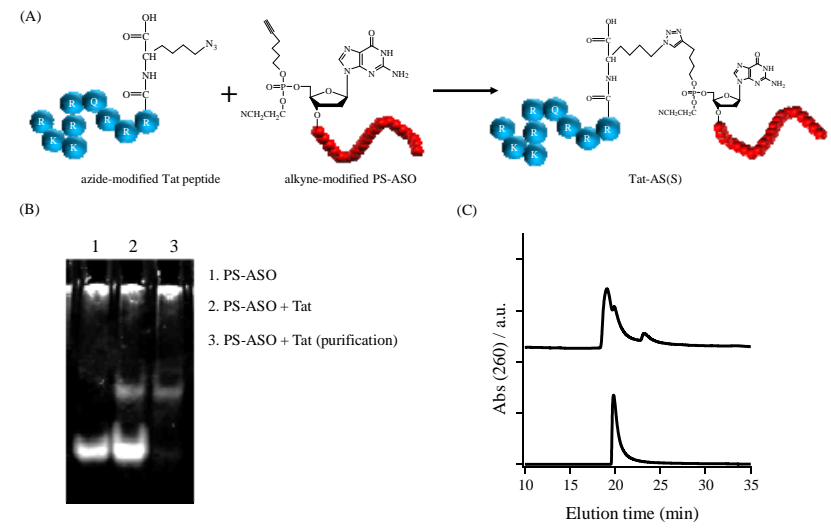

Figure 1. (A) A schematic illustration to prepare the conjugate Tat-AS(S) by Click Chemistry. (B) Confirmation of the conjugation between alkyne-modified PS-ASO and azide-modified Tat peptide, and purification of the conjugate by HPLC fractionation. (C) HPLC chromatograms of alkyne-modified PS-ASO (lower chromatogram) and reaction mixture of alkyne-modified PS-ASO and azide-modified Tat peptide (upper chromatogram). The elution was monitored with UV absorbance at $260 \mathrm{~nm}$.

mixed at high molar ratios compared to PS-ASO, not distinct but smeary bands appeared (data not shown), indicating the formation of the aggregations through electrostatic interactions between cationic Tat peptides and anionic PS-ASOs. The reaction between alkyne and azide groups preferentially occurred at the same molar ratio, while the non-specific interactions defeated the reaction at the other molar ratios. After the reaction at the same molar ratio, the reaction mixture was analyzed with high-performance liquid chromatography (HPLC). From the chromatograms monitored by UV absorbance at $260 \mathrm{~nm}$ before and after the reaction, the peak of PS-ASO shifted to an earlier elution time, indicating the increment in hydrophilicity of PS-ASO by conjugation with Tat peptide (Figure 1C). After fractionation with HPLC and freeze dry, we confirmed the obtained POCPs, denoted by Tat-AS(S), is high purity from no observation of unreacted PS-ASO (Figure 1B). We also obtained the POCs, denoted by Tat-AS(O), and the POCPs with scramble sequence for c-myb, denoted by Tat-SC(S), in a similar manner.

As described above, PS-ODNs are known to have a higher stability in serum than ODNs with phosphodiester backbones. We evaluated the stability of the obtained Tat-AS(O)s and

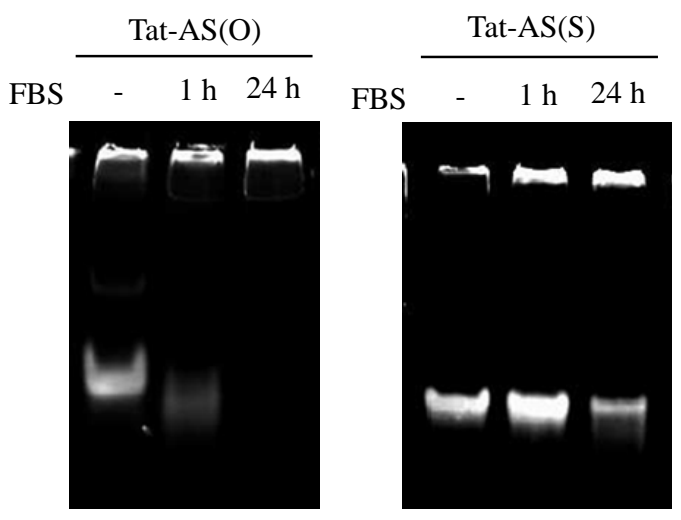

Figure 2. PAGE migration patterns for Tat-AS(O) and Tat-AS(S) after incubation in $90 \%$ of FBS for the indicated times at $37^{\circ} \mathrm{C}$. 


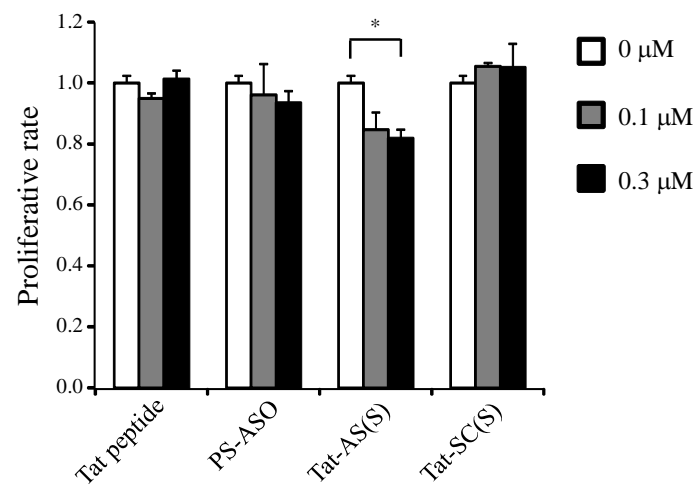

Figure 3. Inhibition of cell proliferation by treatment with Tat peptide, PS-ASO, Tat-AS(S) or Tat-SC(S). Results represent the mean \pm S.D. $(\mathrm{n}=3) . * P<0.01$.

Tat-AS(S)s in $90 \%$ of FBS by PAGE migration patterns. After 1 hour incubation, the band of Tat-AS(S)s did not change (Figure $2)$. On the other hand, the band of Tat-AS(O)s showed faster migration than that of non-treated Tat-AS(O)s, suggesting the partial degradation of Tat-AS(O)s. After 24 hours, half of Tat-AS(S)s were degraded, while Tat-AS(O)s were completely done. In this study, we do not know the degradation of peptide because we observe only ODNs stained with SYBR Gold. The migration of Tat-AS(S)s incubated in serum did not change compared to that of non-treated Tat-AS(S)s, suggesting the Tat peptide conjugated with PS-ODN was also protected from peptidases. The fast migration of Tat-AS(O)s after 1 hour implies that not only ODN but Tat peptide was degraded.

Figure 3 shows the cell proliferation rates after treatment with Tat-AS(S)s. The treatment with Tat-AS(S)s suppressed the cell proliferation of A549 cells, a human alveolar adenocarcinoma cell line, in a dose dependent manner, while that with the naked PS-ASOs did not show any suppression. Tat-SC(S)s also did not show any suppression, indicating that this inhibitory effect was sequence-specifically caused. The result suggests that PS-ASOs bearing Tat peptides can improve the cellular uptake and suppress the target gene expression.

In the field of drug development, the application of Click Chemistry is increasing for exploration of useful compounds. The most of the researches use the reaction concerning the formation of a triazole ring by use of copper catalyst. In this study, we obtained Tat-AS(S)s in a simple manner by mixing alkyne-modified PS-ODN(S)s and azide-modified Tat peptides. PS-ASOs are known to promote the activation of ribonuclease $\mathrm{H}$ (RNase $\mathrm{H})^{16}$ other than high stability in serum. RNase $\mathrm{H}$ recognizes an RNA-DNA heteroduplex and cleaves the RNA strand $^{2}$. As PS-ODNs destabilize the duplex ${ }^{17}$, PS-ASO strand is easy to be released after the cleavage of RNA strand, and hybridize with another target RNA. These facts indicate PS-ASO plays an essential role for knockdown of target gene expression not only out of but in cell. As most drug carriers are incorporated into cells through endocytosis pathway, it is important to design the functions, such as proton-sponge effect, to promote the escape from endosomal vesicles. On the other hand, Tat peptides were considered to enter cells via temperature- and energy-independent, non-endocytic pathway ${ }^{18}$. However, the recent researches using living cells revealed that Tat and other arginine-rich peptides are taken up by cells through macropinocytosis as major routes of internalization ${ }^{19,20}$. Arginine-rich peptides interact with membrane-associated proteoglycan such as heparin sulfate and chondroitin sulfate, resulting in activation intracellular signals to promote macropinocytosis ${ }^{21}$. Short arginine peptides, such as R4 and R8 are likely to follow macropinocytosis pathway, while R12 and R16 are likely to penetrate cellular membranes. Although we do not know the internalization pathway of Tat-AS(S)s into the cells, we would achieve more efficient gene suppression by controlling the length of arginine residues and modification to induce cytoplasmic translocation.

In conclusion, we prepared POCPs using Click Chemistry. Tat-AS(S)s showed a high resistance for enzymatic degradation in serum, and sequence-specifically inhibited the cell proliferation in a dose dependent manner. The present study suggests that Tat-AS(S) can be used for the treatment of cancers and inflammatory diseases by modulating gene expressions.

\section{Acknowledgement}

This work was financially supported by a JST CREST program (15656522).

\section{Supplementary data}

Supplementary data (Materials and Methods) associated with this article can be found, in the online version.

\section{References and notes}

(1) Crooke, S. T. Curr. Mol. Med., 2004, 4, 465.

(2) Stein, H.; Hausen, P. Science 1969, 166, 393.

(3) Zalachoras, I.; Grootaers, G.; van Weert, L. T.; Aubert, Y.; de Kreij, S. R.; Datson, N. A.; van Roon-Mom, W. M.; Aartsma-Rus, A.; Meijer, O. C. BMC Neurosci., 2013, 14, 5.

(4) Stephenson, M. L.; Zamecnik, P. C. Proc. Nat. Acad. Sci. U. S. A., 1978, 75, 285.

(5) Campbell, J. M.; Bacon, T. A.; Wickstrom, E. J. Biochem. Biophys. Methods, 1990, 20, 259.

(6) Margus, H.; Padari, K.; Pooga, M. Mol. Ther., 2012, 20, 525.

(7) Ryser, H. J.; Shen, W. C. Proc. Nat. Acad. Sci. U. S. A., 1978, 75, 3867.

(8) Frankel, A. D.; Pabo, C. O. Cell 1988, 55, 1189.

(9) Brooks, H.; Lebleu, B.; Vives, E. Adv. Drug Deliv. Rev., 2005, 57, 559.

(10) Muratovska, A.; Eccles, M. R. FEBS lett., 2004, 558, 63.

(11) Chaloin, L.; Vidal, P.; Lory, P.; Mery, J.; Lautredou, N.; Divita, G.; Heitz, F. Biochem. Biophys. Res. Commun., 1998, 243, 601.

(12) Antopolsky, M.; Azhayeva, E.; Tengvall, U.; Auriola, S.; Jaaskelainen, I.; Ronkko, S.; Honkakoski, P.; Urtti, A.; Lonnberg, H.; Azhayev, A. Bioconj. Chem., 1999, 10, 598.

(13) Kolb, H. C.; Finn, M. G.; Sharpless, K. B. Angew. Chem. Int. Ed. Engl., 2001, 40, 2004.

(14) Spiteri, C.; Moses, J. E. Angew. Chem. Int. Ed. Engl., 2010, 49, 31.

(15) Wolff, L. Crit. Rev. Oncog., 1996, 7, 245.

(16) Wu, H.; Lima, W. F.; Zhang, H.; Fan, A.; Sun, H.; Crooke, S. T. J. Biol. Chem., 2004, 279, 17181.

(17) Jaroszewski, J. W.; Clausen, V.; Cohen, J. S.; Dahl, O. Nucleic acids Res., 1996, 24, 829.

(18) Vives, E.; Brodin, P.; Lebleu, B. J. Biol. Chem., 1997, 272, 16010.

(19) Richard, J. P.; Melikov, K.; Vives, E.; Ramos, C.; Verbeure, B.; Gait, M. J.; Chernomordik, L. V.; Lebleu, B. J. Biol. Chem., 2003, 278, 585.

(20) Richard, J. P.; Melikov, K.; Brooks, H.; Prevot, P.; Lebleu, B.; Chernomordik, L. V. J. Biol. Chem., 2005, 280, 15300. 
(21) Nakase, I.; Takeuchi, T.; Tanaka, G.; Futaki, S. Adv. Drug Deliv. Rev., 2008, 60, 598. 